\title{
Mortality, readmission, and reoperation after hip fracture in nonagenarians
}

\author{
Jeff Chien-Fu Lin ${ }^{1,2^{*}}$ (D) and Wen-Miin Liang ${ }^{3^{*}}$
}

\begin{abstract}
Background: Osteoporotic hip fractures are associated with high mortality and morbidity in people of advanced age; however, few studies have investigated the complication rates in nonagenarians. In this study, we applied a competing risk analysis to estimate the mortality, readmission, and reoperation rates after surgery for hip fracture among nonagenarians.

Methods: A total of 11,184 nonagenarians (aged $\geq 90$ ) who received surgery for hip fracture during the period 1 January 1997 and 31 December 2010 were selected from Taiwan's National Health Insurance (NHI) database. Nonagenarians were followed up until the end of 2012, death, or the date they left the NHI program. Cumulative mortality was estimated using the Kaplan-Meier analysis and risk factors for mortality were investigated using a Cox proportional hazards model. Competing risk analysis was used to estimate cumulative incidence rates and to assess the risk factors for reoperation and readmission.

Results: The mortality rates were $29.5 \%$ at 1 year, $45.0 \%$ at 2 years and $78.1 \%$ at 5 years. The cumulative incidence rates of reoperation were $7.3 \%$ at 1 year, $9.2 \%$ at 2 years and $11.6 \%$ at 5 years whereas those of readmission were $18.9 \%$ at 1 month and $24.1 \%$ at 3 months. Significant risk factors for death included age, male gender, trochanteric fracture, and higher Charlson comorbidity index (CCI) whereas those for reoperation were age, cervical fracture and higher CCl. Furthermore, age, male gender, and higher CCl were risk factors for readmission.

Conclusions: The overall 2-years mortality rate among nonagenarians in Taiwan was around 45\%, the 2-years reoperation rate was around $9 \%$ and the 90 -days medical complication rate was around $24 \%$. High complication rates are associated with increased risk for death. Postoperative care to prevent medical complications is likely the most effective strategy to reduce mortality rates among nonagenarians with hip fracture.
\end{abstract}

Keywords: Hip fracture surgery, Mortality, Complication, Nonagenarians, Readmission, Reoperation

\section{Background}

Osteoporotic hip fractures are associated with high rates of mortality and morbidity in patients of advanced age, with 1-year mortality rates approaching $30 \%$ in that patient population [1-8]. Although nonagenarians, namely individuals aged 90 years and older, represent a small subgroup, osteoporotic injuries to these individuals pose a great challenge to both surgeons and physicians. One-year mortality rates after hip fracture are as high as $24-50 \%$ in nonagenarians [9-26]. In addition, 1-year readmission rates are $5-30 \%$ and 2-years reoperation rates may be as high as $20 \%$ after surgery for hip fractures in elderly adults

\footnotetext{
* Correspondence: cflin.ntpu@gmail.com; wmliang@mail.cmu.edu.tw ${ }^{1}$ Department of Statistics, National Taipei University, Taipei, Taiwan ${ }^{3}$ Department of Public Health, China Medical University, Taichung, Taiwan Full list of author information is available at the end of the article
}

[27-35]. Unplanned readmissions for medical complications and unplanned reoperations for surgical complications not only increase the mortality but also place an increased socioeconomic burden on healthcare systems and families [27-32, 34, 35]. Previous studies have found that advanced age not only is related to increased mortality but also is associated with increased complication rates after hip fracture [1, 2, 4-8].

The rates of mortality at 1 year after hip fracture in nonagenarians have been shown to range from 24 to $51 \%$ [9-26]. The complication rates after hip fracture in nonagenarians have not been well investigated $[11,15]$. Among the studies that have been conducted, the 2-years complication rate ranged from $30-50 \%[11,15]$. However, the sample sizes of those studies on hip fracture among 
nonagenarians were relatively small $(n=32-919)$ and thus could not precisely quantify all the complication rates after hip fracture among nonagenarians [9-26]. Osteoporotic hip fracture is associated with increased risk of mortality. However, death is a competing risk for complications, which makes it inappropriate to estimate complication rates in the study population with a large proportion of death during the follow-up time using traditional survival analytical methods [36-38]. Most previous studies on long-term complication rates after surgery for hip fracture among nonagenarians did not consider death of these patients as a competing risk, resulting in overestimation of the complication rates [9-26]. Therefore, we applied a competing risk analysis using data from a nationwide population database in Taiwan to estimate the mortality, readmission and reoperation rates after surgery for hip fracture among nonagenarians.

\section{Methods}

\section{Data source and subjects}

Data on patients in this study were retrospectively collected from two sources, namely the National Health Insurance (NHI) Database and the National Register of Deaths Database maintained by Taiwan's Ministry of Health and Welfare. The NHI Database consists of standard computerized claims documents submitted by medical institutions seeking reimbursement through the National Health Insurance (NHI) program from 1996 to the present. The NHI program started in 1995 and as of 2012 covers the medical needs for more than 23 million people, representing more than $99 \%$ of the population of Taiwan. The completeness and accuracy of the NHI database exceed $98-99 \%$ and are guaranteed by Taiwan's Ministry of Health and Welfare and the Bureau of National Health Insurance. All subjects in this study were 90 or more years old (nonagenarians) who received surgery for hip fracture during the period 1 January 1997 - 31 December 2010. As described previously [39], subjects were identified from the database with (a) first discharge disease codes of hip fracture [based on International Classification of Disease, Ninth Revision, Clinical Modification (ICD9-CM) codes 820, 820.0, 820.00, $820.01,820.02,820.09,820.8,820.03,820.2,820.20$, and 820.21 and (b) procedure codes with surgery of internal fixation or hemiarthroplasty (based on ICD9-CM codes $79.15,79.35$, and 81.52). The first admission date for hip fracture was defined as the index day of surgery. The exclusion criteria included subjects with pathological fractures (ICD9-CM codes 733.14 and 733.15) or open hip fractures (ICD9-CM codes 820.1, 820.10, 820.11, 820.12, 820.19 , and 820.9). Subjects who underwent surgery for injuries to the pelvis, femur, or hip region before the index day were also excluded to avoid confounding effects. Comorbidities of each subject were evaluated before or at the time of the index day according to the Charlson comorbidity index (CCI) [40]. All subjects were followed until the end of 2012, exiting the NHI program, or death.

\section{Outcomes of interest}

We estimated three possible outcomes: (a) overall cumulative mortality; (b) cumulative incidence of the first reoperation due to surgical complications, and (c) cumulative incidence of the first medical readmission within 90 days due to medical complications after the index surgery. The duration from the index day to the day of death was defined as the overall survival time. Subjects who were alive at the end of the study or who were lost to follow-up were censored. The duration from the index day to the day of the first postoperative unplanned reoperation for surgical complications was defined as the first reoperation time. The indications for reoperation included conversion to/revision arthroplasty, surgical site infection, removal of implant due to complications, mechanical complication (including loss reduction, screw back out or cutting out, skin irritation, and implant broken/failure), dislocation, avascular necrosis of the femoral head, second hip fracture, and malunion/ nonunion during the follow-up period.

The duration from the index day to the day of the first medical complication within 90 days after the index surgery was defined as the first medical readmission. Complications occurring within 90 days after the index surgery which required extra days of hospital stay or readmission to hospital for treatments included stroke, acute myocardial infarction, pulmonary embolism, acute renal failure, or acute respiratory failure. There is no clear cut-off point between medical complications and newly developed comorbidities after major surgery. We, therefore, interpreted a shorter duration from the index day to the day of the first medical readmission as indicating a greater probability that the readmissions were caused directly or indirectly by the surgery.

\section{Statistical analysis}

The overall survival rates were estimated using the Kaplan-Meier (K-M) analysis. The log-rank test and multivariate Cox's proportional hazards model were applied to estimate the effects of risk factors on survival time. The mortality rate in our cohort was very high; therefore, standard survival analysis was not appropriate for examining the medical and surgical complication rates. An important independent censoring assumption of standard survival analysis implies that patients who are censored at a certain time point should be representative for those still at risk at that point in time. However, because subjects who died could not have any outcomes of interest, the death could not be referred to 
as "censored" for the standard survival analysis. Death is referred to as a "competing risk" for the events of interest other than mortality. When the proportion of deaths is large and increases with follow-up time, the cumulative medical complication and reoperation rates will be overestimated based on the traditional $\mathrm{K}-\mathrm{M}$ procedure. It is important to sufficiently account for the competing risk of death in the analysis. Therefore, we estimated the cumulative incidence of first readmission or reoperation based on the cumulative incidence function of competing risk analysis, in which death was considered as a competing risk to analyze the readmission or reoperation time [36-38]. The Gray's test and the Fine and Gray's model, i.e., a multivariate subdistribution hazards model based on competing risk analysis, were applied to evaluate the effects of risk on readmission and reoperation. The risk factors evaluated in those models included age, sex, fracture type, operation type, and CCI. All data management and analysis were performed using the statistical package SAS 9.4 (SAS Institute, Cary, NC) and R 3.2.2 [available at http://www.R-project.org/, R Development Core Team (2015), R: A language and environment for statistical computing. R Foundation for Statistical Computing, Vienna, Austria, especially the $\mathrm{R}$ libraries survival, cmprsk, and mstate].

\section{Results}

A total of 11,184 nonagenarians who underwent surgery as primary treatment for hip fracture during the period 1997-2010 satisfied the inclusion criteria and were enrolled in the study. The cohort comprised 7,560 (67.6\%) women and 3,624 (32.4\%) men. Among those patients, 4,411 (39.4\%) had a cervical fracture and 6,773 (60.6\%) had a trochanteric fracture. Primary treatment included internal fixation in 7,264 (64.9\%) and hemiarthroplasty in 3,920 (35.1\%) patients (Table 1). Trochanteric fracture was slightly more prevalent than cervical fracture in women. The median survival times were 2.35 years $(95 \%$ CI: $2.28-2.42$ years) for nonagenarians, 2.56 years $(95 \%$ CI: $2.47-2.65$ years) for patients with cervical fracture, and 2.198 years (95\% CI: $2.12-2.288$ years) for patients with trochanteric fracture. The mortality rates were $5.5 \%$ at 1 month, $13.8 \%$ at 3 months, $20.3 \%$ at 6 months, $29.5 \%$ at 1 year, $45.0 \%$ at 2 years, $78.1 \%$ at 5 years and $95.9 \%$ at 10 years. The $1-, 2-, 5-$, and 10 -years cumulative incidence rates of the first reoperation were 7.3, 9.2, 11.6 , and $12.2 \%$, respectively, while those for the first medical readmission at 1 and 3 months were 18.9 and 24.1\%, respectively (Table 2 and Fig. 1). Cumulative mortality and cumulative readmission rates were significantly higher and the cumulative reoperation rate was significantly lower in patients with trochanteric fracture than in those with cervical fracture (Table 2 and Fig. 1).
We evaluated the effects of risk factors on survival using multivariate survival analysis. Age, male gender, trochanteric fracture, and higher CCI were significant risk factors for mortality. For each 1-year increase in age, the hazard ratio (HR) increased by 1.015 (95\% CI: 1.012-1.020). Men had a 31\% (HR, 1.31; 95\% CI: $1.26-$ 1.37) higher risk of overall death than women. Trochanteric fracture had a 1.14 times (95\% CI: 1.06-1.23) higher hazard ratio (HR) of overall death than cervical fracture. Patients with a CCI of one or more were at markedly greater risk for overall death than patients without comorbidities $(\mathrm{CCI}=1$, HR 1.12, 95\% CI: $1.06-$ 1.18; $\mathrm{CCI}=2$, HR 1.20, 95\% CI: $1.13-1.28 ; \mathrm{CCI}=3$, HR 1.30, 95\% CI: $1.20-1.40$; CCI $\geq 4$, HR 1.55, 95\% CI: $1.45-$ 1.66, Table 3).

We evaluated the effects of risk factors on the time to the first reoperation with a multivariate subdistribution proportional hazards model. The results revealed that older age, cervical fracture, and higher CCI were significant risk factors for reoperation. After controlling for age, gender, and comorbidities, trochanteric fracture had a 0.72 times (95\% CI: $0.59-0.89)$ lower sub-HR for reoperation than fixation. We also explored the effects of risk factors on the time to the first readmission due to medical complications and found that age, male gender, and higher CCI score were significant risk factors for readmission within 90 days after surgery (Table 3). Trochanteric fracture had a similar risk for readmission than cervical fracture.

Medical and surgical complications during follow-up are common after treatment for hip fracture, especially in patients of advanced age. In this study, 2,694 (24.1\%) patients had at least one medical complication within 3 months after the index surgery, with the most common complications being acute pulmonary disorders (such as acute exacerbation of chronic obstructive pulmonary disease, 48.37\%; pneumonia, $43.50 \%$; acute respiratory failure, $30.59 \%$ ) and acute renal failure (11.47\%) (Table 4). Of the 2,694 patients who were readmitted for medical complications within 3 months after the index surgery, $36.5 \%$ died within 3 months of readmission (data not shown in the tables).

A total of 1,027 nonagenarians had at least one reoperation within 2 years after the index surgery. Among those who had reoperations within 2 years after the index surgery, most reoperations were caused by second hip fracture $(53.16 \%)$, conversion to/revision anthroplasty (37.39\%), mechanical complication $(26.97 \%$, including loss reduction, screw back out or cutting out, skin irritation, and implant broken/failure), removal of implant (23.66\%), and infection (21.52\%) (Table 4). Of the patients who required a second surgery, $33.7 \%$ died within 1 year after reoperation (data not shown in the tables). 
Table 1 Baseline characteristics of hip fractures among nonagenarians stratified by fracture type

\begin{tabular}{|c|c|c|c|c|c|c|c|c|}
\hline & & Total & & Cervical & & Trochante & & \\
\hline & & $N=11,1$ & & $N=4,411$ & $\overline{(39.4 \%)}$ & $N=6773$ & $(60.6 \%)$ & \\
\hline & & $\bar{N}$ & (\%) & $N$ & (\%) & $N$ & (\%) & $p$-value \\
\hline Age (yrs) & Mean \pm SD & 93 & (2.7) & 93 & (2.4) & 93 & (2.9) & $<0.001$ \\
\hline Gender & Female & 7,560 & (67.6) & 3,022 & $(68.5)$ & 4,538 & $(67.0)$ & 0.098 \\
\hline & Male & 3,624 & (32.4) & 1,389 & $(31.5)$ & 2,235 & $(33.0)$ & \\
\hline Operation Type & Fixation & 7,264 & $(64.9)$ & 758 & $(17.2)$ & 6,506 & $(96.1)$ & $<0.001$ \\
\hline & Hemiarthroplasty & 3,920 & (35.1) & 3,653 & $(82.8)$ & 267 & (3.9) & \\
\hline $\mathrm{CCl}^{\mathrm{a}}$ Score & 0 & 4,671 & (41.8) & 1,865 & $(42.3)$ & 2,806 & $(41.4)$ & 0.049 \\
\hline & 1 & 2,707 & $(24.2)$ & 1,089 & $(24.7)$ & 1,618 & (23.9) & \\
\hline & 2 & 1,580 & (14.1) & 571 & (12.9) & 1,009 & (14.9) & \\
\hline & 3 & 983 & (8.8) & 403 & $(9.1)$ & 580 & (8.6) & \\
\hline & $\geqq 4$ & 1,243 & $(11.1)$ & 483 & (10.9) & 760 & $(11.2)$ & \\
\hline Hypertension & No & 6,017 & (53.8) & 2,318 & $(52.6)$ & 3,699 & $(54.6)$ & 0.033 \\
\hline & Yes & 5,167 & $(46.2)$ & 2,093 & $(47.4)$ & 3,074 & $(45.4)$ & \\
\hline Diabetes Mellitus & No & 9,847 & $(88.0)$ & 3,911 & $(88.7)$ & 5,936 & $(87.6)$ & 0.107 \\
\hline & Yes & 1,337 & (12.0) & 500 & $(11.3)$ & 837 & $(12.4)$ & \\
\hline Heart Disease & No & 8,296 & $(74.2)$ & 3,300 & $(74.8)$ & 4,996 & $(73.8)$ & 0.216 \\
\hline & Yes & 2,888 & $(25.8)$ & 1,111 & $(25.2)$ & 1,777 & $(26.2)$ & \\
\hline Chronic Pulmonary Disease & No & 8,570 & (76.6) & 3,429 & $(77.7)$ & 5,141 & $(75.9)$ & 0.025 \\
\hline & Yes & 2,614 & (23.4) & 982 & $(22.3)$ & 1,632 & $(24.1)$ & \\
\hline Cerebrovascular Disease & No & 9,377 & $(83.8)$ & 3,708 & $(84.1)$ & 5,669 & $(83.7)$ & 0.618 \\
\hline & Yes & 1,807 & $(16.2)$ & 703 & (15.9) & 1,104 & $(16.3)$ & \\
\hline Cancer & No & 10,353 & (92.6) & 4,069 & $(92.2)$ & 6,284 & $(92.8)$ & 0.302 \\
\hline & Yes & 831 & $(7.4)$ & 342 & $(7.8)$ & 489 & $(7.2)$ & \\
\hline Primary solid cancer & No & 10,397 & $(93.0)$ & 4,082 & $(92.5)$ & 6,315 & $(93.2)$ & 0.162 \\
\hline & Yes & 787 & $(7.0)$ & 329 & $(7.5)$ & 458 & $(6.8)$ & \\
\hline Chronic Liver Disease & No & 10,756 & $(96.2)$ & 4,231 & $(95.9)$ & 6,525 & $(96.3)$ & 0.267 \\
\hline & Yes & 428 & (3.8) & 180 & $(4.1)$ & 248 & (3.7) & \\
\hline Chronic Renal Disease & No & 10,782 & (96.4) & 4,232 & $(95.9)$ & 6,550 & $(96.7)$ & 0.038 \\
\hline & Yes & 402 & (3.6) & 179 & $(4.1)$ & 223 & (3.3) & \\
\hline
\end{tabular}

SD standard deviation

${ }^{a}$ Charlson Comorbidity Index

\section{Discussion}

To the best of our knowledge, this is the first population study to use competing risk analysis to explore mortality and readmission due to medical complications as well as reoperation due to surgical complications after hip fracture among nonagenarians based on data from a nationwide database. A number of meta-analyses have revealed that age is an important risk factor for death in elderly adults after hip fracture $[4,6,7]$. In this study, we investigated the outcomes of 11,184 nonagenarians after hip fracture surgery in Taiwan. We found that the mortality rates were $5.5 \%$ at 1 month, $13.8 \%$ at 3 months, $20.3 \%$ at 6 months, $29.5 \%$ at 1 year, $45.0 \%$ at 2 years, $78.1 \%$ at 5 years, and $95.9 \%$ at 10 years after surgery. Previous studies reported that the 1-, 3-, 6-months, and 1-year mortality rates ranged from 5.9 to $26.4 \%, 15$ to $36 \%, 24.7$ to $42 \%$, and 24 to $51 \%$, respectively [9-26]. The shortterm mortality rates in Taiwan were lower than those in New Zealand, Spain, Scotland, Netherlands, Canada, and the United States; but higher than those in Japan and Korea [9-26]. Previous studies reported that the shortterm mortality rates among nonagenarians varied from region to region [9-26]. Japan, Korea, and Taiwan seem to have lower short-term mortality rates after hip fracture among nonagenarians than those of Western countries. However, the sample sizes in the previous studies were relatively small (from 32 to 919 nonagenarians) compared to that in our study [9-26]. 
Table 2 Cumulative mortality rates and cumulative incidence of first reoperation or readmission after surgery for hip fracture among nonagenarians stratified by fracture type

\begin{tabular}{|c|c|c|c|c|c|c|c|c|c|}
\hline \multirow[b]{3}{*}{ Time } & \multirow{2}{*}{\multicolumn{3}{|c|}{ Mortality }} & \multicolumn{6}{|c|}{ Cumulative Incidence } \\
\hline & & & & \multicolumn{3}{|c|}{$\begin{array}{l}\text { Reoperation } \\
\text { (Surgical Complication) }\end{array}$} & \multicolumn{3}{|c|}{$\begin{array}{l}\text { Readmission } \\
\text { (Medical Complication) }\end{array}$} \\
\hline & Total & Cervical & Trochanteric & Total & Cervical & Trochanteric & Total & Cervical & Trochanteric \\
\hline 1-month & $5.50 \%$ & $4.60 \%$ & $6.10 \%$ & $2.80 \%$ & $4.10 \%$ & $2.00 \%$ & $18.90 \%$ & $18.30 \%$ & $19.20 \%$ \\
\hline 3-month & $13.80 \%$ & $12.50 \%$ & $14.60 \%$ & $4.50 \%$ & $5.90 \%$ & $3.50 \%$ & $24.10 \%$ & $22.90 \%$ & $24.90 \%$ \\
\hline 6-month & $20.30 \%$ & $18.50 \%$ & $21.60 \%$ & $5.60 \%$ & $7.10 \%$ & $4.60 \%$ & & & \\
\hline 1-year & $29.50 \%$ & $27.10 \%$ & $31.10 \%$ & $7.30 \%$ & $9.00 \%$ & $6.20 \%$ & & & \\
\hline 2-year & $45.00 \%$ & $41.80 \%$ & $47.10 \%$ & $9.20 \%$ & $10.80 \%$ & $8.10 \%$ & & & \\
\hline 5-year & $78.10 \%$ & $75.80 \%$ & $79.60 \%$ & $11.60 \%$ & $13.50 \%$ & $10.30 \%$ & & & \\
\hline 10-year & $95.90 \%$ & $95.90 \%$ & $95.90 \%$ & $12.20 \%$ & $14.30 \%$ & $10.90 \%$ & & & \\
\hline
\end{tabular}

Differences in findings among the studies from different countries might be due to differences in selection criteria, distributions of gender-stratified nonagenarians in the populations, distributions of smoking status, physical activity, bone mineral density, nutrition, hip strength, and comorbidities, in addition to sample sizes.

In a review on hip fracture-related mortality among elderly adults from 1996 to 1998 , Haleem et al. found that the rates of mortality ranged from 11 to $23 \%$ at 6 months and from 22 to $29 \%$ at 1 year [3]. In a meta-analysis of mortality after hip fracture in patients of advanced age, Abrahamsen et al. found that the crude mortality rates ranged from 3.3 to $17.2 \%$ at 1 month, 6.4 to $20.4 \%$ at 3 months, 7.1 to $23 \%$ at 6 months, and 5.9 to $59 \%$ at 1 year [4]. They also found that excess mortality was highest in the first 6 months and that it decreased after 1 year, but remained high throughout the next several years. Several studies have shown that hip fractures affected short-term but not long-term mortality among elderly adults $[1,8,12,13,24,41]$. However, others have shown that there is a gradual increase in mortality for up to 5 years after fracture [1, 41-46]. The 1-, 2-, 5years, and 10-years mortality rates were 29.5, 45.0, 78.1 , and $95.9 \%$ in this study. We found that the first-year mortality of nonagenarians after hip fracture was similar to that of elderly adults [1-8]. However, after the first year, the mortality rates increased more rapidly among nonagenarians after hip fracture than those among other elderly age groups in other studies $[15,26]$. Nonagenarians, due to their advanced age, naturally have a very high mortality rate, irrespective of any past history of hip fracture. Whether excess mortality caused by hip fracture among nonagenarians increases persistently for several years after hip fracture remains unclear. We believe that hip fracture and aging both continue to have effects on mortality 1 year after fracture among nonagenarians.
The results of our multivariate survival analysis showed that age, male gender, and higher CCI were significant predictors of mortality after hip fracture in nonagenarians. In a meta-analysis, $\mathrm{Hu}$ et al. reported that the yearly HR for age was 1.05 among elderly adults after hip fracture [7]. In our study, the estimated yearly HR for age was 1.015 (95\% CI: 1.012-1.020). The age effect on mortality in nonagenarians was not as high as in elderly adults. Several previous studies also did not find that age was a risk factor for mortality among nonagenarians [12, 17]. One possible reason for the discrepancy is that our patient population ( $>90$ years) was more homogeneous than the populations included in other population-based studies ( $>65$ years) on predictors of mortality after fracture.

We found that men were at $31 \%$ greater risk for mortality than women (HR, 1.31, 95\% CI: 1.26-1.37). Many meta-analyses have found that elderly men are at higher risk for mortality than elderly women after hip fracture $[4,6,7]$. Hu et al. estimated that men had a $70 \%$ greater risk of overall death than females (HR 1.70; 95\% CI: 1.51-1.04) [7]. Although several studies have reported that male nonagenarians have higher mortality rates than their female counterparts $[16,17,22,26]$, other studies have shown that male gender is not a significant predictor of mortality among nonagenarians [12, 20, 25]. In our study, the HR of male gender in nonagenarians was not as high as that of other age subgroups of elderly adults. Males had more comorbidities, specifically chronic obstructive pulmonary disease, than females in our study. The higher prevalence of multiple comorbidities in males may explain the higher risk. However, in a number of population-based studies, men had higher mortality rates and died earlier than women; therefore, nonagenarians represent a more homogeneous population in which more healthy males survived compared with the younger elderly adults. This explains, at least in part, why the effect of male gender in nonagenarians 


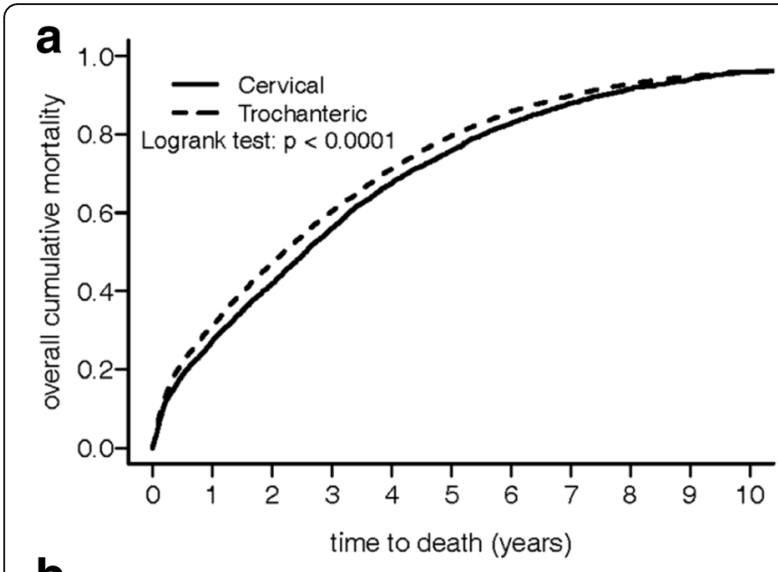

b

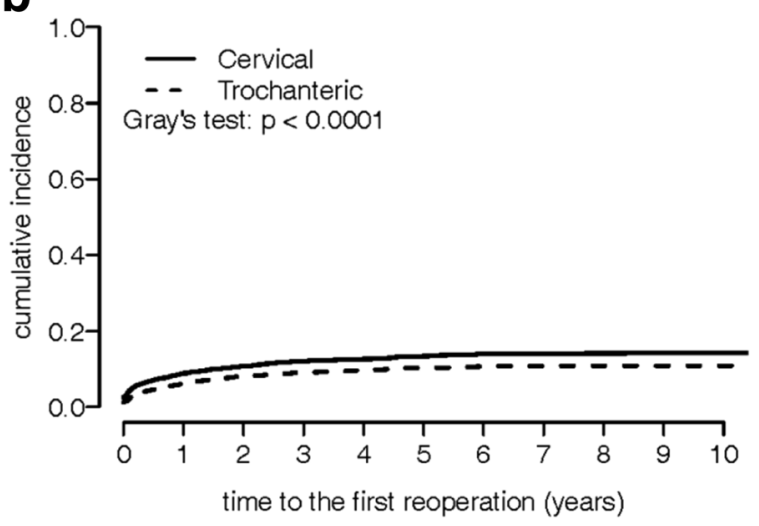

C

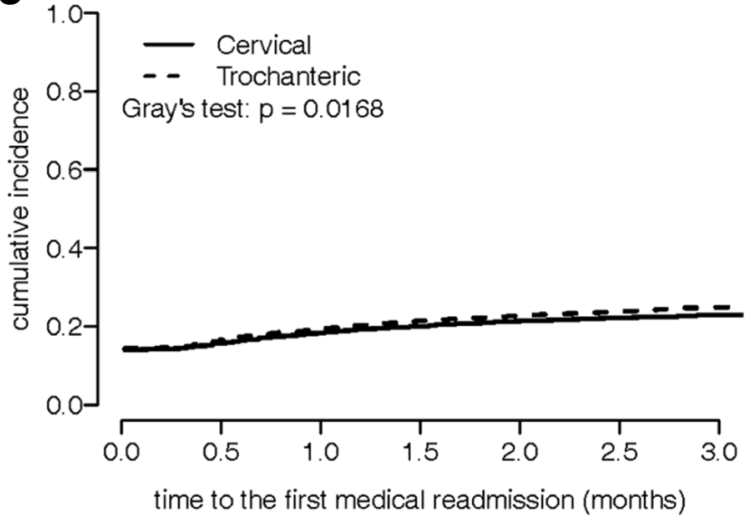

Fig. 1 Ten-year cumulative incidence curve of (a) mortality stratified by fracture type, (b) first reoperation stratified by fracture type, (c) 3months cumulative incidence curves of first medical readmission stratified by fracture type

was not as high as that in the younger elderly subgroups, and may further suggest why some studies did not find that male gender was a predictor of mortality after hip fracture among nonagenarians. The small sample sizes and shorter follow-up durations might also have contributed to some of the differences in the previous studies.

We found that trochanteric fracture was associated with a 14\% greater likelihood of death than cervical fracture (HR, 1.14: 95\% CI: 1.06-1.23). Previous studies on elderly adults after hip fracture have also reported that trochanteric fracture is associated with higher risk for mortality than cervical fracture [2, 47-52]. However, the association between fracture type and mortality in nonagenarians is not consistent among studies [11, 12, 14, 17, 20, 22, 23, 25]. Most studies on hip fracture in nonagenarians have reported that mortality rates are similar between trochanteric fracture and cervical fracture [11, 12, 17, 20, 23, 25]. Only Ishida et al. and Kang et al. demonstrated that trochanteric fracture was associated with a higher risk for mortality than cervical fracture among nonagenarians [14, 22]. In this study, patients with trochanteric fracture were older and nonagenarians had more comorbidities than those reported in studies on younger elderly adults. Gender and age may have a mixed effect on risk of trochanteric fracture.

We found no significant difference between internal fixation and arthroplasty in mortality among nonagenarians, a finding consistent with that reported by Takamine et al. and Tay et al. [20, 25]. However, Ishida et al. demonstrated that hemiarthroplasty was a significant risk factor for mortality among 74 nonagenarians [14]. In our study, most $(82.8 \%)$ patients with cervical fracture received hemiarthroplasty and most (96.1\%) with trochanteric fracture received internal fixation. Natural causes of death among nonagenarians and unbalanced mixed subject-variable distributions between types of operations and fractures all contributed to the reasons for the non-significant differences between internal fixation and arthroplasty for mortality.

In this study, higher CCI was associated with higher risk of mortality among nonagenarians. Several studies have explored the relationship between comorbidities and mortality after hip fracture among nonagenarians and many of the results varied from study to study $[15,16,19,20,26]$. Two studies found that a larger number of comorbidities increased mortality [16, 19] and three studies reported no association between comorbidities and mortality after hip fracture among nonagenarians $[15,20,26]$. Many previous studies have explored whether American Society of Anesthesiologist (ASA) score is predictive of mortality after hip fracture among nonagenarians, but the results were not conclusive [12, 14-18, 22, 23, 25]. Some studies found that a higher ASA score was associated with mortality $[12,14,16,18]$. However, other studies did not find that ASA score was associated with mortality after hip fracture among nonagenarians [15, 17, 22, 23, 25]. Aging, higher CCI and higher ASA scores are all associated with a larger number of and more severe comorbidities. Differences in competing risk of death, confounding variables, such as age, CCI, ASA score, and sample size, may interact with one another within studies resulting in different conclusions among studies about the effects of comorbidities. However, there is still no agreement among researchers as to how 
Table 3 (a) Hazard ratios of the risk factors associated with death from cause-specific hazard model based on multivariate Cox's model, (b) subdistribution hazard ratio of the risk factors associated with the first reoperation, and (c) readmission based on multivariate Fine and Gray's (subdistribution hazard ratio, sub-HR) model from competing risk analysis

\begin{tabular}{|c|c|c|c|c|c|c|c|c|c|c|}
\hline & & \multicolumn{3}{|c|}{ (a) Death } & \multicolumn{3}{|c|}{ (b) Reoperation } & \multicolumn{3}{|c|}{ (c) Readmission } \\
\hline & & HR & (95\%C.I.) & $p$-value & sub-HR & (95\%C.I.) & $p$-value & sub-HR & (95\%C.I.) & $p$-value \\
\hline Age & Year & 1.015 & $(1.012-1.019)$ & $<0.001$ & 0.98 & $(0.95-0.99)$ & 0.041 & 1.02 & $(1.01-1.03)$ & 0.003 \\
\hline \multirow[t]{2}{*}{ Gender } & Female & (REF) & & & (REF) & & & (REF) & & \\
\hline & Male & 1.31 & $(1.26-1.37)$ & $<0.001$ & 0.92 & $(0.82-1.04)$ & 0.182 & 1.71 & $(1.59-1.84)$ & $<0.001$ \\
\hline \multirow[t]{2}{*}{ Fracture } & Cervical & (REF) & & & (REF) & & & (REF) & & \\
\hline & Trochanteric & 1.14 & $(1.06-1.23)$ & $<0.001$ & 0.72 & $(0.59-0.89)$ & 0.002 & 1.08 & $(0.95-1.22)$ & 0.257 \\
\hline \multirow[t]{2}{*}{ Operation } & Fixation & (REF) & & & (REF) & & & (REF) & & \\
\hline & Hemiarthroplasty & 1.03 & $(0.96-1.11)$ & 0.371 & 0.96 & $(0.78-1.18)$ & 0.665 & 1.00 & $(0.88-1.14)$ & 0.998 \\
\hline \multirow[t]{5}{*}{$\mathrm{CCl}^{\mathrm{a}}$} & 0 & (REF) & & & (REF) & & & (REF) & & \\
\hline & 1 & 1.12 & $(1.06-1.18)$ & $<0.001$ & 0.94 & $(0.82-1.08)$ & 0.383 & 2.63 & $(2.37-2.91)$ & $<0.001$ \\
\hline & 2 & 1.20 & $(1.13-1.28)$ & $<0.001$ & 0.97 & $(0.82-1.14)$ & 0.698 & 3.02 & $(2.71-3.37)$ & $<0.001$ \\
\hline & 3 & 1.30 & $(1.20-1.40)$ & $<0.001$ & 1.06 & $(0.87-1.29)$ & 0.570 & 3.39 & $(2.99-3.85)$ & $<0.001$ \\
\hline & 4 & 1.55 & $(1.45-1.66)$ & $<0.001$ & 0.80 & $(0.65-0.97)$ & 0.025 & 3.60 & (3.15-4.13) & $<0.001$ \\
\hline
\end{tabular}

${ }^{\mathrm{a} C h a r l s o n}$ Comorbidity Index

comorbidities should be measured and how the measurement should be evaluated statistically.

Postoperative readmission and reoperation rates increase the use of healthcare resources and are often indicative of healthcare quality for hip fractures. Major medical complications result in readmission, whereas major surgical complications result in reoperation. Several studies on outcomes after surgery for hip fracture in patients $\geq 65$ years revealed that the readmission rate within 3 months varied from 10 to $20 \%$, the 1 -year reoperation rate varied from 7 to $13 \%$ and the 2 -years reoperation rate varied from 15 to $28 \%$ [27-32, 34, 35]. To the best of our knowledge, only two studies have reported on the readmission and reoperation rates after hip fracture among nonagenarians $[11,15]$. Those studies found that the 2-years complication rates were as high as $30-50 \%[11,15]$. Osteoporotic hip fracture, aging, and comorbidities all contributed to excess mortality. If subjects died, these subjects could not have any complications after their death. Readmission or reoperation rate cannot be estimated without considering competing death, which may cause overestimation of the risk, especially for nonagenarians. Using traditional Kaplan-Meier method, the cumulative medical complication and reoperation rates would be overestimated. Kaplan-Meier estimates are biased because the death rate is large and will increase with the follow-up time. Therefore, to obtain a more valid estimate of the risk for medical or surgical complication, we applied a competing risk analysis to estimate the cumulative incidence of the readmission and reoperation [36,37]. We found that the 1-, 2-, and 5-years cumulative incidence rates of the first reoperation were $7.3,9.2$, and $11.6 \%$ for the nonagenarians, respectively. Moreover, the 1- and 3months cumulative incidence rates of the first medical readmission were 18.9 and $24.1 \%$, respectively, for the nonagenarians in Taiwan. Because competing death was not considered, previous studies had higher long-term reoperation rates than those of the current study $[36,37]$. Our finding implies that nonagenarians appeared to have a higher all-cause hazard ratio for death, had lower risks for various surgical complications when we considered the competing death in our analysis. Therefore, nonagenarians had a slightly lower reoperation rate than those in the previous studies. However, even under this circumstance, male nonagenarians still had slightly higher shortterm readmission rates of medical complications. The competing risk of death had less effect on the 3-months readmission rates than on the long-term reoperation rates. Nonagenarians did not die quickly due to aging during the 3-months follow-up after hip fracture. Therefore, nonagenarians in our study had similar short-term readmission rates after hip fracture as nonagenarians in the previous studies.

In this study, predictors of mortality included older age, male gender, trochanteric fracture, and higher CCI score. However, trochanteric fracture correlated with a low risk for reoperation (Table 3). After controlling for age, gender, operation type, and comorbidities, we found that patients with trochanteric fracture were at $14 \%$ greater risk for death than those with cervical fracture $(\mathrm{HR}=1.14,95 \% \mathrm{CI}: 1.06-1.23)$ but that their risk of reoperation was $28 \%$ lower than patients with cervical fracture $(\mathrm{HR}=0.72$; 95\% CI: 0.59-0.89). This finding implies that nonagenarians with trochanteric fracture appeared to have a higher all-cause hazard for death but 


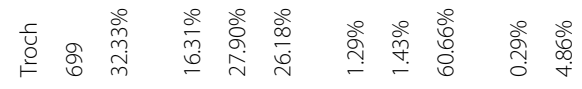

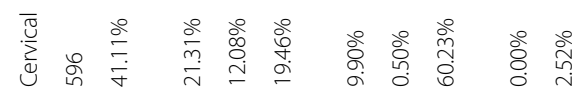

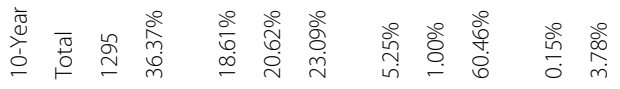

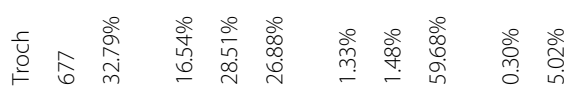

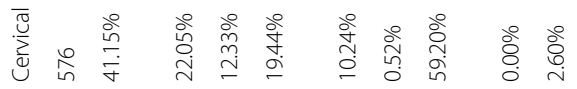

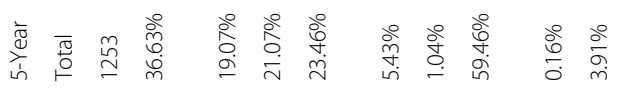

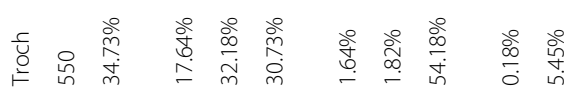

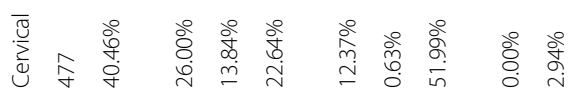

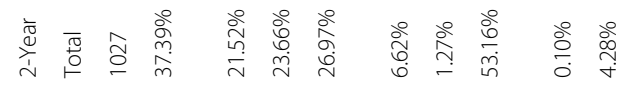

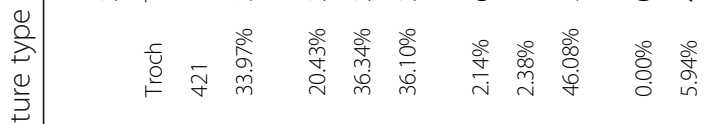

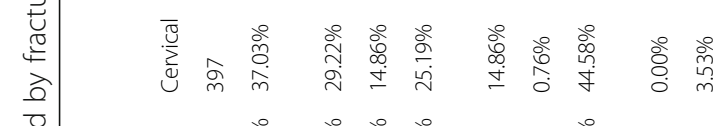

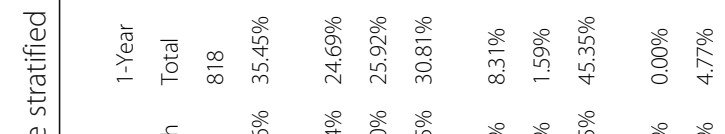

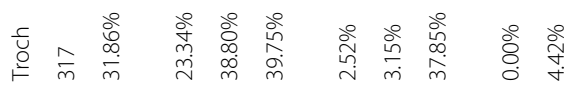

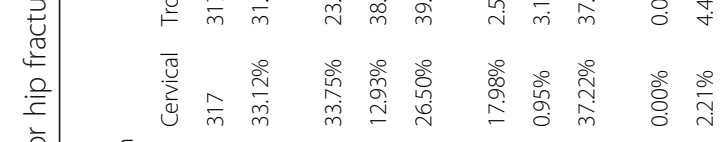

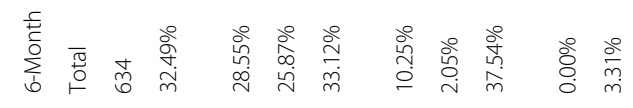

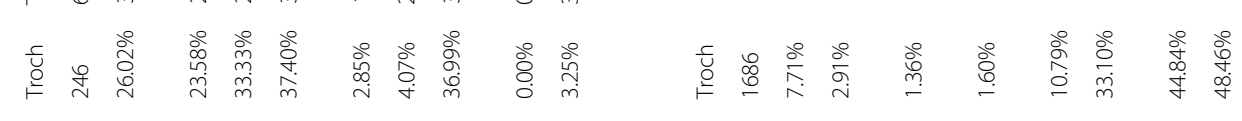

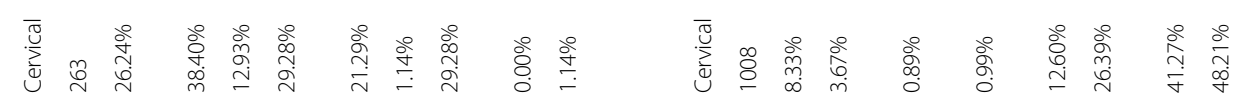

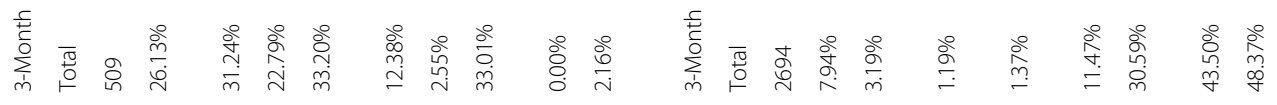

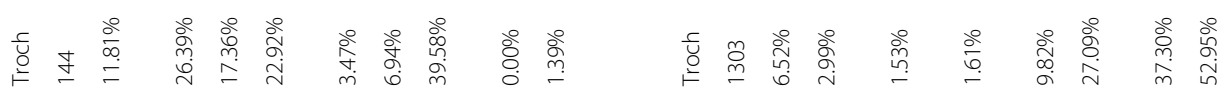

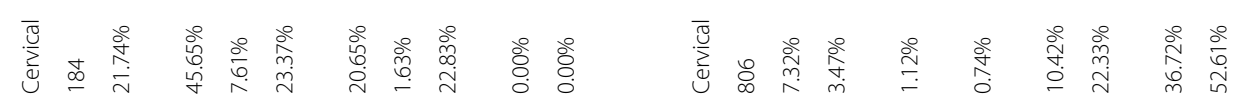

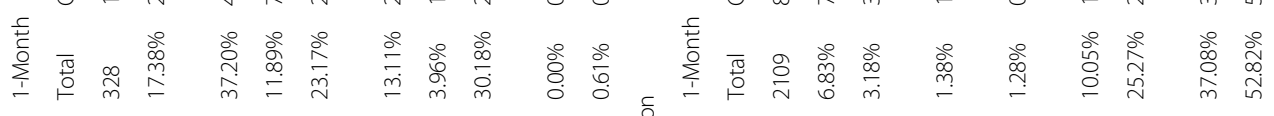


lower risks of reoperation than those with cervical fracture. When the follow-up time was short, the cumulative mortality rate was still low; many subjects with trochanteric fracture still had a higher hazard ratio for 90-days readmission (sub-HR $=1.08$ ) than those with cervical fracture. As described in the previous study [39], during the first 3-months follow-up, the short-term death rate was small, subjects with trochanteric fracture had larger readmission hazard rate (sub-HR $=1.08$ ) than subjects with cervical fracture. In contrast, after 6-months follow-up, there were fewer subjects with trochanteric fracture during follow-up but they were healthier and therefore exposed to less risk for long-term surgical complications. Thus, subjects with trochanteric fracture had a smaller risk (sub-HR $=0.72$ ) for surgical reoperation than those with cervical fracture. When a population had much higher mortality rate than complication rate during the early phase of follow-up, using high cost but more benefit treatments to reduce the long-term surgical complication would be limited. When the competing hazards of a risk factor on mortality and complication are different, it raises the difficulty of conducting analysis and interpretation. A cost-effective analysis from a competing risk viewpoint may be quite different from that without considering the competing death. In practice, competing risk analysis is a useful tool to address the actual risk and to provide a more realistic result to make a decision.

The competing death phenomenon not only affected the effect of fracture type, but also slightly affected other factors, such as age, gender, and operation type, during long-term follow-up after surgery in our study. Moreover, the competing death phenomenon may have no effect on short-term follow-up after surgery. As older age, male gender, trochanteric fracture, and higher CCI were significant risk factors for mortality, age, male gender, and higher CCI also had higher risks for medical readmission within the 3-months follow-up period after surgery (Table 3 ).

Most readmissions occur within 3 months [30, 35]. Some studies also found that readmissions or reoperations were associated with higher mortality within 1 year $[27,28,31,32,34,35]$. We found that $984(36.5 \%)$ of 2,694 nonagenarians died within 3 months after the first medical complications and that 859 (33.7\%) of 1,296 nonagenarians died within 1 year after the first reoperation. Jennings and de Boer found that 12 (37.5\%) of 32 nonagenarians who had surgical complications died within 6 months, and 17 (38.6\%) of 44 who had medical complications died within 6 months [11]. Our analysis of nonagenarians revealed similar findings to those of previous studies on elderly adults after hip fractures [11, 28, 31-34]. Prevention of hip fracture among nonagenarians is likely the best way to reduce the high risk effect of aging on mortality, readmission, and reoperation after surgery.

The overall 3-months, 1 - and 5-years mortality rates among nonagenarians in Taiwan were around 14, 30 and $78 \%$, respectively. The 1 - and 5 -years reoperation rates were around 7 and 12\%, respectively. The 30- and 90days medical complication rates were around 19 and $24 \%$, respectively. Once nonagenarians had the first readmission, $36.5 \%$ died within 3 months after medical complications. Once nonagenarians had the first reoperation, 33.7\% died within 1 year after reoperation (data not shown in the tables). The impact of medical complications on mortality among the nonagenarians was extraordinarily high. Therefore, postoperative care to prevent medical complications should be the most effective strategy to reduce the mortality rates among nonagenarians with hip fracture. However, prevention of hip fracture in this patient population is the best way to reduce mortality, readmission and reoperation after surgery.

\section{Limitations}

All of the patients in this study were nonagenarians who underwent surgical treatment for hip fracture; however, there was great variation in follow-up period among the patients (2-11 years). The NHRI database differs from the hip fracture registry database in that the former does not record all clinical information [2, 53]. Therefore, some unknown clinical confounding factors might have existed or changed during the follow-up period. In addition, we did not adjusted for many of the risk variables, such as general condition prior to surgery, smoking and alcohol behavior, BMI, bone mineral density, comorbidities, and quality of life. Agreement about how comorbidities should be evaluated and how the measures of comorbidities should be placed into a statistical model is still absent. Aging, higher ASA cores, delayed surgery, and higher $\mathrm{CCI}$ are associated with one another. For example, a poor ASA score is caused by a large number of comorbidities, which implies that more time is needed to stabilize the medical comorbidities, and further suggests a long waiting time for surgery, as well as poor outcomes after surgery. We used the CCI to represent the combined severity of multiple comorbidities, which has been shown to be a key consideration in hip fracture $[54,55]$. In addition, we used ICD9-CM codes to define the medical complications and reasons for reoperation, which might have lacked the precision needed for a precise evaluation of the estimated readmission and reoperation rates. Finally, the readmission rates and reoperation rates might also vary depending on the definitions used. Therefore, caution should be taken in extrapolating our results. 


\section{Conclusions}

During the period 1997-2010, 11,184 nonagenarians received surgery for hip fracture in Taiwan. The 3months postoperative mortality and medical readmission rates were 13.8 and $23.8 \%$, respectively, and the 2 -years mortality and reoperation rates were 45.0 and $9.2 \%$, respectively. Among nonagenarians who were readmitted for the first time, $36.5 \%$ died within 3 months. Among nonagenarians who received their first reoperation, $33.7 \%$ died within 1 year. High complication rates caused excess mortality. Postoperative care to prevent medical complications is an important strategy to reduce the mortality rates in nonagenarians with hip fracture.

\section{Abbreviations}

ASA: American Society of Anesthesiologists; CCl: Charlson comorbidity index; HR: Hazard ratio; ICD9-CM: International classification of disease, ninth revision, clinical modification; KM: Kaplan-Meier; NHI: National health insurance; sub-HR: subdistribution hazard ratio

\section{Acknowledgements}

We would like to thank Mr. Peter Wilds and Mr. Jeffrey Conrad for their assistance in providing professional writing services.

\section{Funding}

This study was supported in part by grants from the China Medical University (CMU102-PH-01) and China Medical University Hospital (DMR-106-155). This study was also supported in part by Taiwan Ministry of Health and Welfare Clinical Trial Center (MOHW106-TDU-B-212-113004), China Medical University Hospital.

\section{Availability of data and materials}

These databases were composed of anonymous secondary data released to the public for research purposes. The instruction of permission to access the database is shown in the website of the Taiwan's Ministry of Health and Welfare at http://www.mohw.gov.tw/.

\section{Authors' contributions}

JCFL participated in the study conception and design, literature search, data analyses and interpretation, and drafting of the manuscript. WML participated in the literature search, data analysis and interpretation, and revision of the manuscript. All authors had access to the data and take responsibility for the integrity of the data and the accuracy of the data analysis. Both authors read and approved the final manuscript.

\section{Competing interests}

The authors declare that they have no competing interests.

\section{Consent for publication}

Not applicable.

\section{Ethical approval and consent to participate}

National Health Insurance (NHI) Database and the National Register of Deaths Database were administrated by Taiwan's Ministry of Health and Welfare. The consent from the subjects was waived since all subjects' data had been encrypted using the same encryption algorithm to cross-link the data while protecting the privacy of the subjects. The study protocol was approved by the institutional review board (IRB) of China Medical University Hospital (protocol \# CMUH102-REC2-012).

\section{Publisher's Note}

Springer Nature remains neutral with regard to jurisdictional claims in published maps and institutional affiliations.

\section{Author details}

${ }^{1}$ Department of Statistics, National Taipei University, Taipei, Taiwan. ${ }^{2}$ Department of Orthopedic Surgery, Wan Fang Hospital, Taipei Medical University, Taipei, Taiwan. ${ }^{3}$ Department of Public Health, China Medical University, Taichung, Taiwan.

Received: 18 May 2016 Accepted: 20 March 2017

Published online: 04 April 2017

\section{References}

1. Tsuboi M, Hasegawa $Y$, Suzuki S, Wingstrand $H$, Thorngren KG. Mortality and mobility after hip fracture in Japan: a 10-years follow-up. J Bone Joint Surg Br. 2007:89:461-6.

2. Holt G, Smith R, Duncan K, Finlayson DF, Gregori A. Early mortality after surgical fixation of hip fractures in the elderly: an analysis of data from the scottish hip fracture audit. J Bone Joint Surg Br. 2008;90:1357-63.

3. Haleem S, Lutchman L, Mayahi R, Grice JE, Parker MJ. Mortality following hip fracture: trends and geographical variations over the last 40 years. Injury. 2008;39:1157-63.

4. Abrahamsen B, van Staa T, Ariely R, Olson M, Cooper C. Excess mortality following hip fracture: a systematic epidemiological review. Osteoporos Int. 2009;20:1633-50.

5. Brauer CA, Coca-Perraillon M, Cutler DM, Rosen AB. Incidence and mortality of hip fractures in the United States. JAMA. 2009:302:1573-9.

6. Haentjens P, Magaziner J, Colon-Emeric CS, et al. Meta-analysis: excess mortality after hip fracture among older women and men. Ann Intern Med. 2010;152:380-90.

7. Hu F, Jiang C, Shen J, Tang P, Wang Y. Preoperative predictors for mortality following hip fracture surgery: a systematic review and meta-analysis. Injury. 2012:43:676-85.

8. Wang CB, Lin CF, Liang WM, et al. Excess mortality after hip fracture among the elderly in Taiwan: a nationwide population-based cohort study. Bone. 2013:56:147-53.

9. MacCollum 3rd MS, Karpman RR. Approaches to senior care \#8. Hip fractures in nonagenarians. Orthop Rev. 1989;18:471-7.

10. Keene GS, Parker MJ, Pryor GA. Mortality and morbidity after hip fractures. BMJ. 1993:307:1248-50.

11. Jennings $A G$, de Boer P. Should we operate on nonagenarians with hip fractures? Injury. 1999:30:169-72.

12. Alarcon T, Gonzalez-Montalvo JI, Barcena A, Saez P. Further experience of nonagenarians with hip fractures. Injury. 2001:32:555-8.

13. Shah MR, Aharonoff GB, Wolinsky P, Zuckerman JD, Koval KJ. Outcome after hip fracture in individuals 90 years of age and older. J Orthop Trauma. 2001; 15:34-9.

14. Ishida Y, Kawai S, Taguchi T. Factors affecting ambulatory status and survival of patients 90 years and older with hip fractures. Clin Orthop Relat Res. 2005:436:208-15.

15. Ooi LH, Wong $\mathrm{TH}$, Toh $\mathrm{CL}$, Wong HP. Hip fractures in nonagenarians-a study on operative and non-operative management. Injury. 2005;36:142-7.

16. Holt G, Macdonald D, Fraser M, Reece AT. Outcome after surgery for fracture of the hip in patients aged over 95 years. J Bone Joint Surg Br. 2006;88:1060-4.

17. van de Kerkhove MP, Antheunis PS, Luitse JS, Goslings JC. Hip fractures in nonagenarians: perioperative mortality and survival. Injury. 2008:39:244-8.

18. Holt G, Smith R, Duncan K, Hutchison JD, Gregori A. Outcome after surgery for the treatment of hip fracture in the extremely elderly. J Bone Joint Surg Am. 2008;90:1899-905.

19. Bergeron E, Moore L, Fournier K, Gravel C, Lavoie A. Patients with isolated hip fracture must be considered for surgery irrespectively of their age, comorbidity status and provenance: a statement applicable even to nonagerians. Arch Orthop Trauma Surg. 2009;129:1549-55.

20. Takamine B, Morii T, Watanabe H, Tajima T, Satomi K. Short-term postoperative mortality events in patients over 80 years of age with hip fracture: analysis at a single institution with limited medical resources. J Orthop Sci. 2010;15:437-42.

21. Clement ND, Aitken SA, Duckworth AD, McQueen MM, Court-Brown CM. The outcome of fractures in very elderly patients. J Bone Joint Surg Br. 2011; 93:806-10.

22. Kang BJ, Lee YK, Lee KW, Won SH, Ha YC, Koo KH. Mortality after hip fractures in nonagenarians. J Bone Metab. 2012;19:83-6. 
23. Kadowaki M, Kono M, Nishiguchi K, Kakimaru H, Uchio Y. Mortality in patients with hip fracture aged over 90 years: a report from a progressively aging island. Arch Gerontol Geriatr. 2012;54:e113-7.

24. de Leur K, Vroemen JP, Vos DI, Elmans L, van der Laan L. Outcome after osteosynthesis of hip fractures in nonagenarians. Clin Interv Aging. 2014;9:41-9.

25. Tay YW, Hong CC, Murphy D. Functional outcome and mortality in nonagenarians following hip fracture surgery. Arch Orthop Trauma Surg. 2014;134:765-72.

26. Kapicioglu M, Ersen A, Saglam Y, Akgul T, Kizilkurt T, Yazicioglu O. Hip fractures in extremely old patients. J Orthop. 2014;11:136-41.

27 Boockvar KS, Halm EA, Litke A, et al. Hospital readmissions after hospital discharge for hip fracture: surgical and nonsurgical causes and effect on outcomes. J Am Geriatr Soc. 2003;51:399-403.

28 French DD, Bass E, Bradham DD, Campbell RR, Rubenstein LZ. Rehospitalization after hip fracture: predictors and prognosis from a national veterans study. J Am Geriatr Soc. 2008;56:705-10.

29 Radcliff TA, Henderson WG, Stoner TJ, Khuri SF, Dohm M, Hutt E. Patient risk factors, operative care, and outcomes among older community-dwelling male veterans with hip fracture. J Bone Joint Surg Am. 2008;90:34-42.

30 Giusti A, Barone A, Razzano M, Pizzonia M, Oliveri M, Pioli G. Predictors of hospital readmission in a cohort of 236 elderly discharged after surgical repair of hip fracture: 1-year follow-up. Aging Clin Exp Res. 2008;20:253-9.

31 Hahnel J, Burdekin H, Anand S. Re-admissions following hip fracture surgery. Ann R Coll Surg Engl. 2009;91:591-5.

32 Khan MA, Hossain FS, Dashti Z, Muthukumar N. Causes and predictors of early re-admission after surgery for a fracture of the hip. J Bone Joint Surg Br. 2012:94:690-7.

33 Khan MA, Hossain FS, Ahmed I, Muthukumar N, Mohsen A. Predictors of early mortality after hip fracture surgery. Int Orthop. 2013;37:2119-24.

34 Buecking B, Eschbach D, Koutras C, et al. Re-admission to level 2 unit after hipfracture surgery - risk factors, reasons and outcome. Injury. 2013:44:1919-25.

35 Teixeira A, Trinquart L, Raphael M, Bastianic T, Chatellier G, Holstein J. Outcomes in older patients after surgical treatment for hip fracture: a new approach to characterise the link between readmissions and the surgical stay. Age Ageing. 2009;38:584-9.

36 Berry SD, Ngo L, Samelson EJ, Kiel DP. Competing risk of death: an important consideration in studies of older adults. J Am Geriatr Soc. 2010:58:783-7.

37 Pintilie M. Competing risks : a practical perspective. Chichester, England. Hoboken, NJ: John Wiley \& Sons; 2006.

38 Beyersmann J. Competing risks and multistate models with R. New York: Springer; 2011.

39 Lin JC, Liang WM. Mortality and complications after hip fracture among elderly patients undergoing hemodialysis. BMC Nephrol. 2015;16:100.

40 Charlson ME, Pompei P, Ales KL, MacKenzie CR. A new method of classifying prognostic comorbidity in longitudinal studies: development and validation. J Chronic Dis. 1987;40:373-83.

41 Johnell O, Kanis JA. An estimate of the worldwide prevalence, mortality and disability associated with hip fracture. Osteoporos Int. 2004;15:897-902.

42 Magaziner J, Lydick E, Hawkes W, et al. Excess mortality attributable to hip fracture in white women aged 70 years and older. Am J Public Health. 1997;87:1630-6.

43 Kanis JA, Oden A, Johnell O, De Laet C, Jonsson B, Oglesby AK. The components of excess mortality after hip fracture. Bone. 2003;32:468-73.

44 Forsen L, Sogaard AJ, Meyer HE, Edna T, Kopjar B. Survival after hip fracture: short- and long-term excess mortality according to age and gender. Osteoporos Int. 1999;10:73-8.

45 Vestergaard P, Rejnmark L, Mosekilde L. Increased mortality in patients with a hip fracture-effect of pre-morbid conditions and post-fracture complications. Osteoporos Int. 2007;18:1583-93.

46 Gronskag AB, Romundstad P, Forsmo S, Langhammer A, Schei B. Excess mortality after hip fracture among elderly women in Norway. The HUNT study. Osteoporos Int. 2012;23:1807-11.

47 Lu-Yao GL, Baron JA, Barrett JA, Fisher ES. Treatment and survival among elderly Americans with hip fractures: a population-based study. Am J Public Health. 1994;84:1287-91.

48 Muraki S, Yamamoto S, Ishibashi H, Nakamura K. Factors associated with mortality following hip fracture in Japan. J Bone Miner Metab. 2006;24:100-4.

49 Haentjens $\mathrm{P}$, Autier $\mathrm{P}$, Barette $\mathrm{M}$, et al. Survival and functional outcome according to hip fracture type: a 1-year prospective cohort study in elderly women with an intertrochanteric or femoral neck fracture. Bone. 2007:41: 958-64.
50 Lin WP, Wen CJ, Jiang CC, Hou SM, Chen CY, Lin J. Risk factors for hip fracture sites and mortality in older adults. J Trauma. 2011;71:191-7.

51 Kesmezacar H, Ayhan E, Unlu MC, Seker A, Karaca S. Predictors of mortality in elderly patients with an intertrochanteric or a femoral neck fracture. J Trauma. 2010;68:153-8.

52 Castronuovo E, Pezzotti P, Franzo A, Di Lallo D, Guasticchi G. Early and late mortality in elderly patients after hip fracture: a cohort study using administrative health databases in the Lazio region, Italy. BMC Geriatr. 2011;11:37.

53 Gjertsen JE, Vinje T, Engesaeter LB, et al. Internal screw fixation compared with bipolar hemiarthroplasty for treatment of displaced femoral neck fractures in elderly patients. J Bone Joint Surg Am. 2010;92:619-28.

54 Radley DC, Gottlieb DJ, Fisher ES, Tosteson AN. Comorbidity risk-adjustment strategies are comparable among persons with hip fracture. J Clin Epidemiol. 2008;61:580-7.

55 Kirkland LL, Kashiwagi DT, Burton MC, Cha S, Varkey P. The Charlson comorbidity index score as a predictor of 30-days mortality after hip fracture surgery. Am J Med Qual. 2011;26:461-7.

\section{Submit your next manuscript to BioMed Central and we will help you at every step:}

- We accept pre-submission inquiries

- Our selector tool helps you to find the most relevant journal

- We provide round the clock customer support

- Convenient online submission

- Thorough peer review

- Inclusion in PubMed and all major indexing services

- Maximum visibility for your research

Submit your manuscript at www.biomedcentral.com/submit
) Biomed Central 